\title{
On the Asymptotic Enumeration of LEGO Structures
}

\author{
Mikkel Abrahamsen and Søren Eilers
}

CONTENTS

1. Introduction

2. Results and Conjectures

3. Methods

Acknowledgments

References
2000 AMS Subject Classification: 05A16, 05-04

Keywords: asymptotic enumeration, LEGO, Monte Carlo methods
We investigate experimentally the growth regimes of the number of LEGO structures that can be constructed contiguously from $n$ blocks of equal shape and color.

\section{INTRODUCTION}

\subsection{Background and Overview}

The number of LEGO structures that can be constructed contiguously from $n$ blocks of equal shape and color is an interesting but rather elusive combinatorial object. With the number $T_{b \times w}(n)$ defined as the count of all contiguous structures made of $n$ LEGO blocks of size $b \times w$, identified up to rotation in the $X Y$-plane and translation, a value such as $T_{2 \times 4}(10)$ is unknown, although it is only, in all likelihood, a 17-digit number. The lack of apparent recursive structure in the problem leaves us little combinatorial machinery to understand and compute such numbers.

Our interest in these quantities stems from the observation by the second-named author that a claim made by the LEGO Group for decades to the effect that $T_{2 \times 4}(6)=$ 102981500 is false (indeed, only maximal-height towers such as shown as in Figure 1(a) were counted). In synchronized but independent efforts, the authors computed the correct value $T_{2 \times 4}(6)=915103765$. However, our interest has shifted away from such individual values toward an attempt to understand the asymptotic behavior of each integer sequence $\left(T_{b \times w}(n)\right)_{n \in \mathbb{N}}$, and how the asymptotics vary with the dimension $b \times w$.

Even this remains shrouded in mystery. We know only that certain associated sequences grow supermultiplicatively, and hence that quantities

$$
h_{b \times w}=\lim _{n \rightarrow \infty} \sqrt[n]{T_{b \times w}(n)}
$$

are well defined. Furthermore, it was proved in [Durhuus and Eilers 05] that $78.32 \leq h_{2 \times 4} \leq 176.58$.

The main results presented here provide experimental evidence supporting the conjectures:

- $T_{b \times w}(n)$ grows as $h_{b \times w}^{n} \cdot n^{-3 / 2}$ (Conjecture 2.1). 


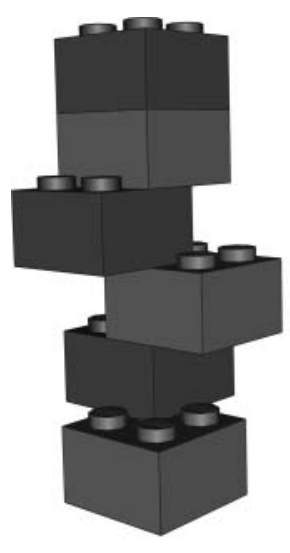

(a)

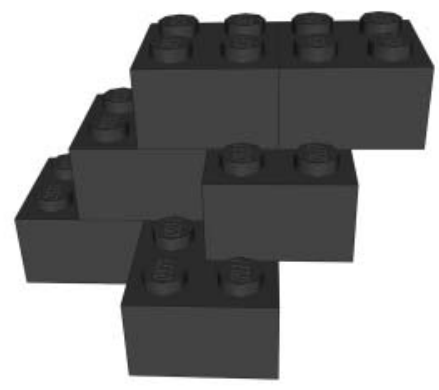

(b)

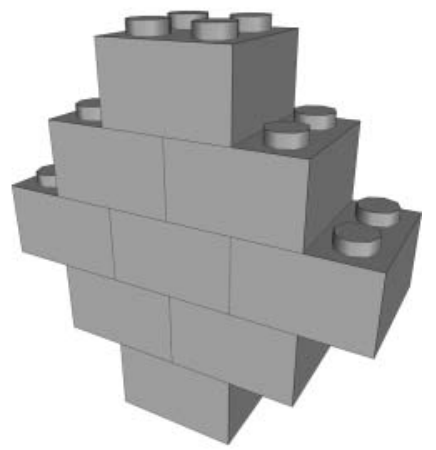

(c)

FIGURE 1. Examples of LEGO structures.

- $h_{b \times w}$ varies with $(b, w)$ by a quadratic expression (Conjecture 2.2).

As described in [Abrahamsen and Eilers 07], significant speedups in the algorithms for exact counts are available. However, since run times grow exponentially in $n$, we are able to compute more than ten terms in only a few of these sequences, even though we have allowed computing times up to $450 \mathrm{CPU}$ hours on an otherwise idle mainframe computer. Thus we have taken to Monte Carlo methods to count far enough to give qualified input to a theoretical approach to understanding the asymptotics of sequences such as $\left(T_{b \times w}(n)\right)_{n \in \mathbb{N}}$. These methods are described in Section 3.2 below.

\subsection{Notation and Conventions}

We study numbers $T_{b \times w}(n)$ defined as the count of all contiguous structures made of $n$ LEGO blocks of size $b \times w$, identified up to rotation and translation (a mathematical definition of this concept is given in [Durhuus and Eilers 05], but it is the same as what any LEGO user, mathematician or otherwise, would employ). The set of all these equivalence classes is denoted by $\mathcal{T}_{b \times w}[n]$.

The number

$$
k_{b \times w}=(2 b-1)(2 w-1)+\left(1-\delta_{b, w}\right)(b+w-1)^{2}
$$

counts the number of ways one block of a given dimension may be added to another.

\section{RESULTS AND CONJECTURES}

When the method is fixed, the computing times for numbers $T_{b \times w}(n)$ by exact methods are roughly proportional to the values found. Rather surprisingly, although we can give a partial explanation in Remark 3.3 below, the time consumption for applying our Monte Carlo methods of Section 3.2 varies dramatically in a nonobvious way with the dimensions, as indicated in Figure 2 (left). The figure illustrates the reciprocal of the number of experiments required to compute a $95 \%$ confidence interval for $T_{b \times w}(20)$ of a length that is less than $1 \%$ of the estimated value; thus high bars indicate dimensions that are especially prone to such an analysis. As indicated, dimensions $1 \times w$ are particularly convenient to work with, but taking into account that a variation of sizes is desirable in this setting, we have selected dimensions $1 \times 2,1 \times 14$, $4 \times 5$, and $7 \times 7$ among these dimensions for further study.

As mentioned in the introduction, our theoretical knowledge concerning the growth of $T_{b \times w}(n)$ is very limited, and needless to say, an experimental approach to such a problem can lead to only circumstantial evidence. Furthermore, the analysis is rather sensitive to imprecisions in the data. Consider, however, Figure 2 (right), which has been obtained by plotting

$$
T_{b \times w}(n+1) / T_{b \times w}(n) \cdot\left(T_{b \times w}(14) / T_{b \times w}(13)\right)^{-1}
$$

for $b \times w \in\{1 \times 2,1 \times 14,4 \times 5,7 \times 7\}$. The values for $T_{b \times w}(n)$ have been computed by our Monte Carlo method with confidence intervals only $1 \%$ of the observed value. The plots are consistent with a growth regime that is 

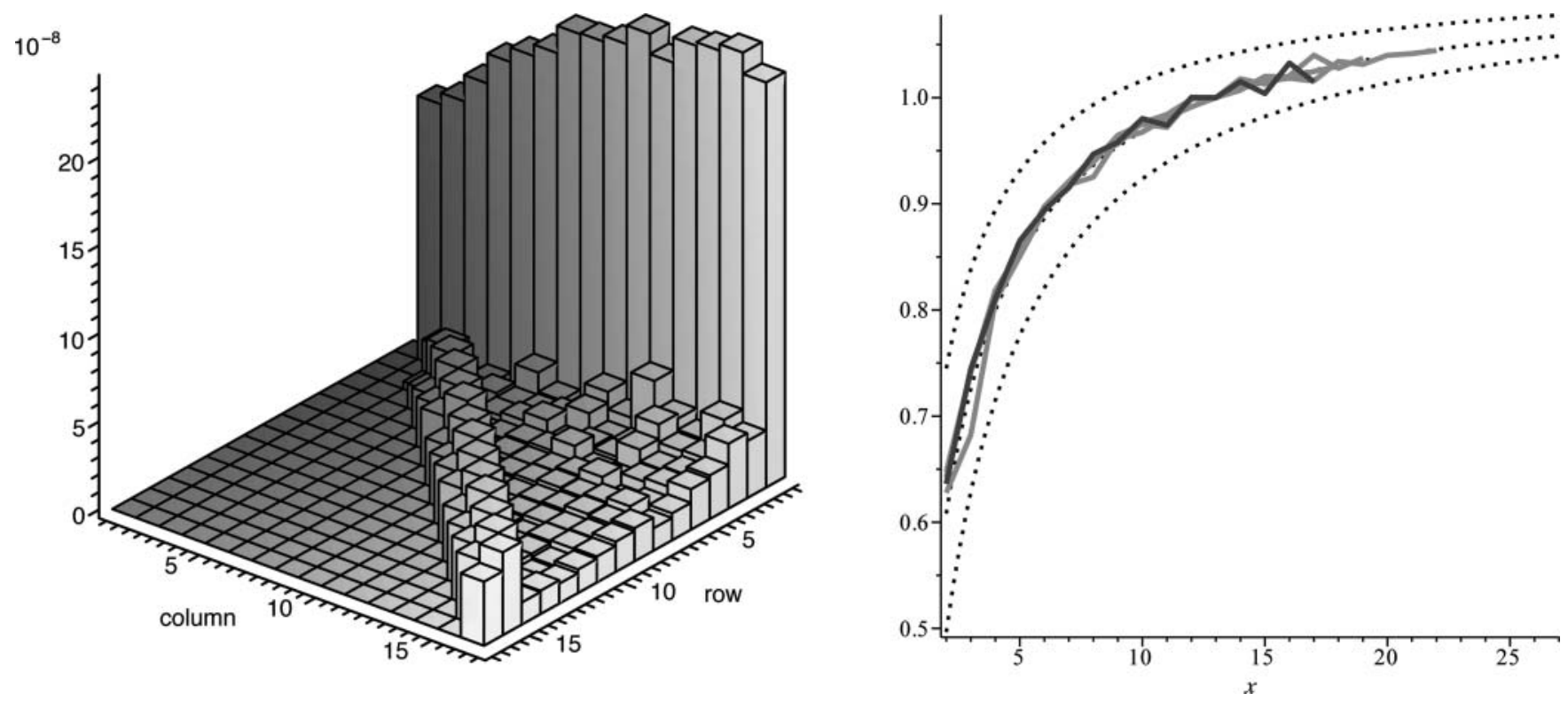

FIGURE 2. Left: Inverse computing times for our Monte Carlo method as a function of the block size. Right: Growth regimes associated with various block sizes. Dotted lines (from below) correspond to subexponential terms of order $n^{-1}$, $n^{-3 / 2}$, and $n^{-2}$, respectively.

roughly $H^{n-1} \cdot n^{-3 / 2}$, but far from conclusive in nature. Somewhat more carefully, we make the following conjecture:

Conjecture 2.1. $T_{b \times w}(n) \sim C H^{n-1} n^{P}$ for suitably chosen $C=c_{b \times w}, H=h_{b \times w}$, and $P=p_{b \times w}$.

\subsection{Growth Constants and Their Variation}

Based on Conjecture 2.1 and extensive experimentation, we have found estimates for $c_{b \times w}, h_{b \times w}$, and $p_{b \times w}$ for $b, w$ with $1 \leq b \leq w \leq 16$, as indicated in Figure 3 and tabulated in [Abrahamsen and Eilers 07]. These estimates have been achieved by a least-squares approximation of the form

$$
C+H(n-1)+P \log (n)
$$

to a semilogarithmic plot of approximated values of $T_{b \times w}(5), \ldots, T_{b \times w}(20)$, generated by our Monte Carlo methods with a confidence interval at most $10 \%$ of the size of the estimated value.

We have analyzed these estimates as indicated in Figure 4. The graphs show how well the observed entropies may be approximated by a quadratic fit, as indicated both globally and on certain contours. The case of square blocks requires special attention, but only in the requirement that the fitting quadratic expression approximate twice the observed entropies. Similarly, the observed value for the constant term $C$ in the square case is half of the observed value in the nonsquare case.

Conjecture 2.2. There exists a function

$$
p(w, b)=A w^{2}+B b w+C b^{2}+D w+E b+F
$$

that approximates growth constants to a high level of accuracy as follows:

$$
p(w, b) \sim h_{b \times w}, \quad p(w, w) \sim 2 h_{w \times w} .
$$

We estimate

$$
\begin{aligned}
p(w, b)= & 13.68 b w+2.92 b^{2}+3.29 w^{2}-12.62 b \\
& -8.89 w+4.23
\end{aligned}
$$

Note that our formula (1-1) for $k_{b \times w}$ provides a trivial lower bound for $h_{b \times w}$ of quadratic order. We note, with a sketch of the proof postponed to Section 3, that an upper bound of this type also exists:

Theorem 2.3. We have, for any $w>1$ and any $1<b<w$,

$$
\begin{aligned}
h_{1 \times w} & \leq 7 w^{2}+7 w-14, \\
h_{w \times w} & \leq 18 w^{2}, \\
h_{b \times w} & \leq 24 w^{2}+36 b w-48 w .
\end{aligned}
$$



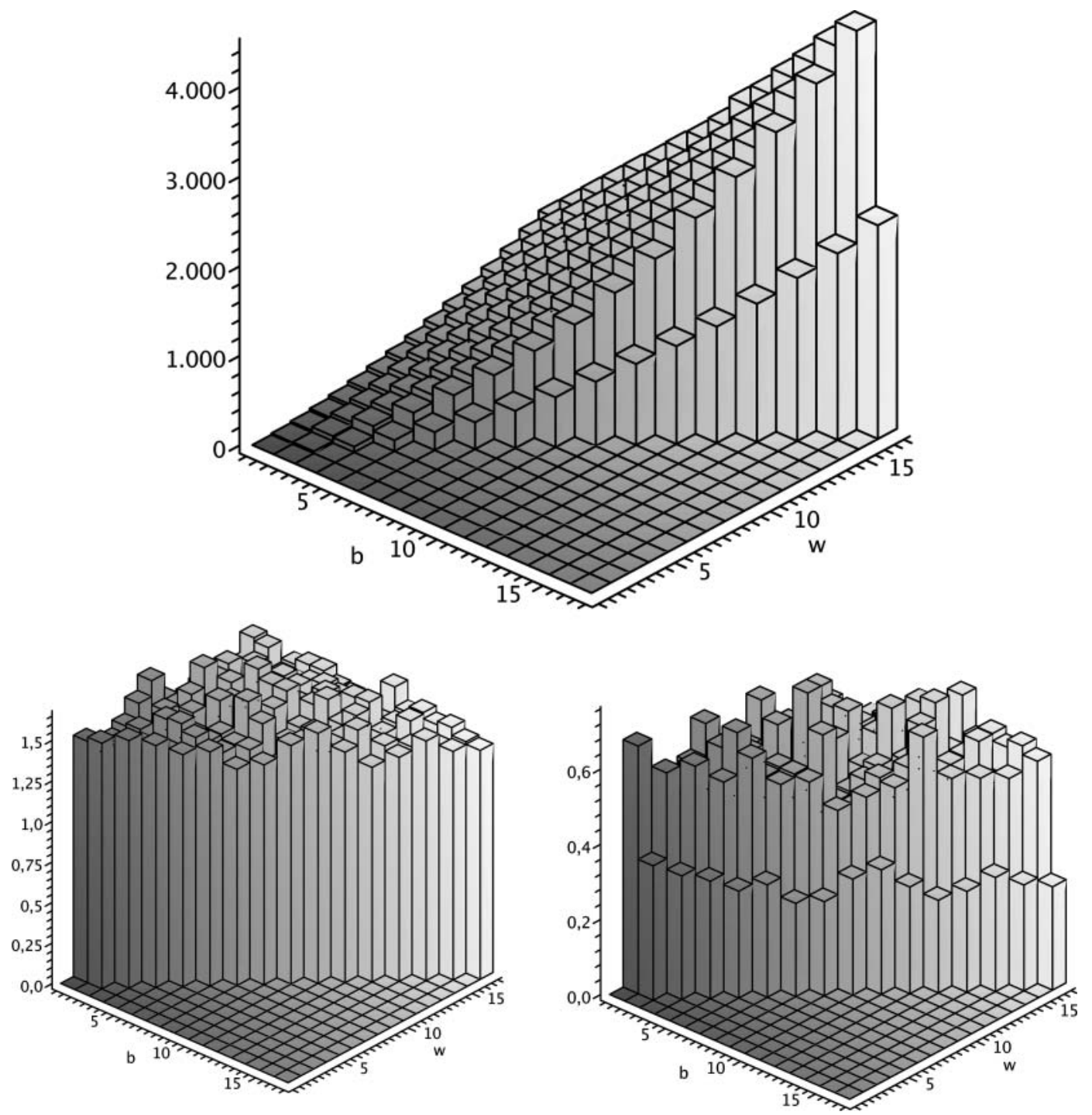

FIGURE 3. Estimated values for $h_{b \times w}$ (top), $-p_{b \times w}$ (bottom left), and $c_{b \times w}$ (bottom right).

\subsection{Subexponential Growth}

A subexponential growth of the order $n^{-3 / 2}$ is consistent with the observations in [Madras 95, p. 682] for 3dimensional lattice animals, lattice trees, etc., but it must be admitted that our observations are too imprecise to warrant a firm conclusion that this is indeed the correct exponent.

It is perhaps interesting to note that if one limits attention to flat LEGO structures, where all blocks are aligned in a plane as in Figure 1(c), the subexponential growth is clearly of a higher order (see [Abrahamsen and Eilers 07] for further details). Since our Monte Carlo method does not work well in this case, we are unable to verify that the correct order, as predicted by [Madras 95], is $n^{-1}$.

Although the objects considered are not trees in any sense, the appearance of the power $-3 / 2$ is indicative of a generating function with a square-root singularity at $1 / h_{b \times w}$, which is often seen in counting problems associated with trees; see, e.g., [Flajolet and Sedgewick 08]. Thus it is a natural approach to try to understand the numbers $T_{b \times w}(n)$ using models based on trees.

However, if this were possible, one would expect that as regards trees, the average height of a structure with $n$ blocks of a fixed size universally grow as $\sqrt{n}$. This turns out not to be the case, as quite clearly indicated by Monte Carlo experiments. We have the following conjecture. 

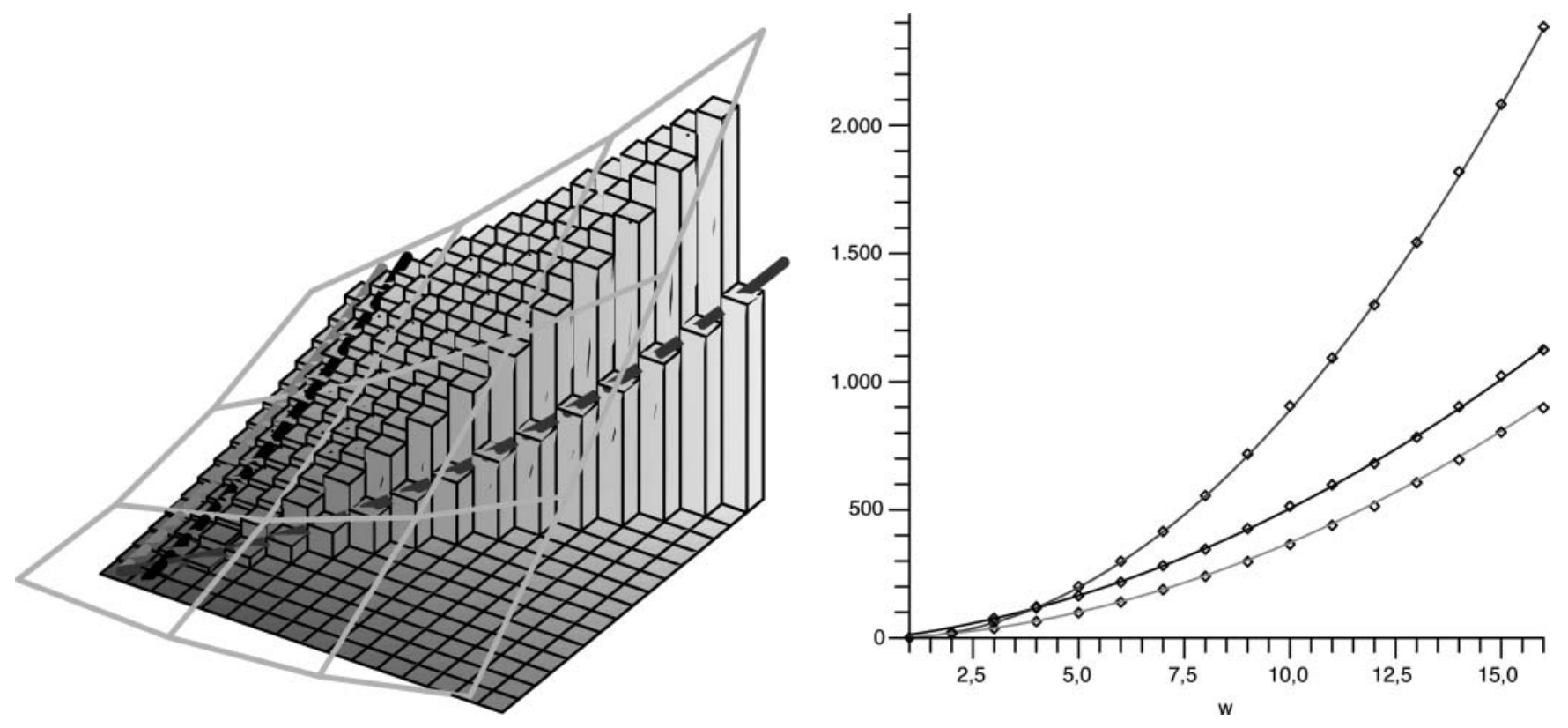

FIGURE 4. Left: Growth constants with quadratic fit. Right: Fits specialized to dimensions (from below) $1 \times w, 2 \times w$, and $w \times w$.

Conjecture 2.4. The average height of elements in $\mathcal{T}_{b \times w}[n]$ grows as a power $n^{\alpha}$ with $\alpha$ in the range $[0.6,0.7]$.

The exponent $\alpha$ varies considerably with the block size, as indicated in Figure 5. It is interesting to note that although LEGO's features allow one to place blocks from below, thus not contributing to the height, the restrictions on the options for building upward force higher averages than for $(1, \ldots, m)$-trees, where $m$ is the maximal number of blocks that may be placed on top of another. The phenomenon is more distinctive for small blocks, corresponding to the fact that in such cases, there are very few different ways to construct low buildings.

\section{METHODS}

\subsection{Overcounting Strategy}

We follow a strategy developed in [Durhuus and Eilers 11] and recall a few concepts from that paper. The set of all $k_{b \times w}$ ways to attach one block of a fixed dimension on top of another is called the set of positions. A partition of this set is a subdivision into $b \times w$ sets $\mathcal{P}_{1}, \ldots, \mathcal{P}_{b w}$, one for each stud of the block, with the extra property that all positions in $\mathcal{P}_{i}$ employ stud $i$. We found in [Durhuus and Eilers 05] that a partition of the 46 positions on a $2 \times 4$ block into a partition with

$$
46=16+15+7+5+2+1+0+0
$$

elements gives optimal estimates. However, in order to give estimates valid for any block size, and to simplify our Monte Carlo method described below, we shall focus entirely on even subdivisions such as

$$
46=8+8+8+8+8+6+0+0,
$$

which minimize the following quantities:

Definition 3.1. The term count $\mathrm{T}$ of a partition is the number of its nonempty sets. The element count $\mathrm{E}$ is the maximal number of elements in a set in the partition.

More precisely, we aim to minimize first $\mathrm{T}$ and then $\mathrm{E}$ for the minimal $\mathrm{T}$.

For a fixed partition with term and element counts $\mathrm{T}$ and $\mathrm{E}$, respectively, we define $\mathcal{U}_{b \times w}[n]$ as the collection of $(2 \boldsymbol{T}+(n-2)(2 \boldsymbol{T}-1))$-tuples with entries in $\{0,1, \ldots, E\}$ such that precisely $n-1$ of the entries are nonzero. As in [Durhuus and Eilers 05], a surjective map

$$
\Psi: \mathcal{U}_{b \times w}[n] \rightarrow \mathcal{T}_{b \times w}[n] \cup\{\text { FAIL }\}
$$

may be defined by reading off, in a systematic way, from the nonzero entries of the tuple how to attach blocks to reach a structure. The first $2 T$ entries specify what to attach to the base block $\mathrm{B}_{b \times w}$ by reading each nonzero integer as an instruction to place a block in one of the at most $\mathrm{E}$ positions on one of the $\mathrm{T}$ selected studs (or corresponding holes) on either side of the block. The added blocks will be enumerated $1,2, \ldots$, and the next $2 \mathrm{~T}-1$ 

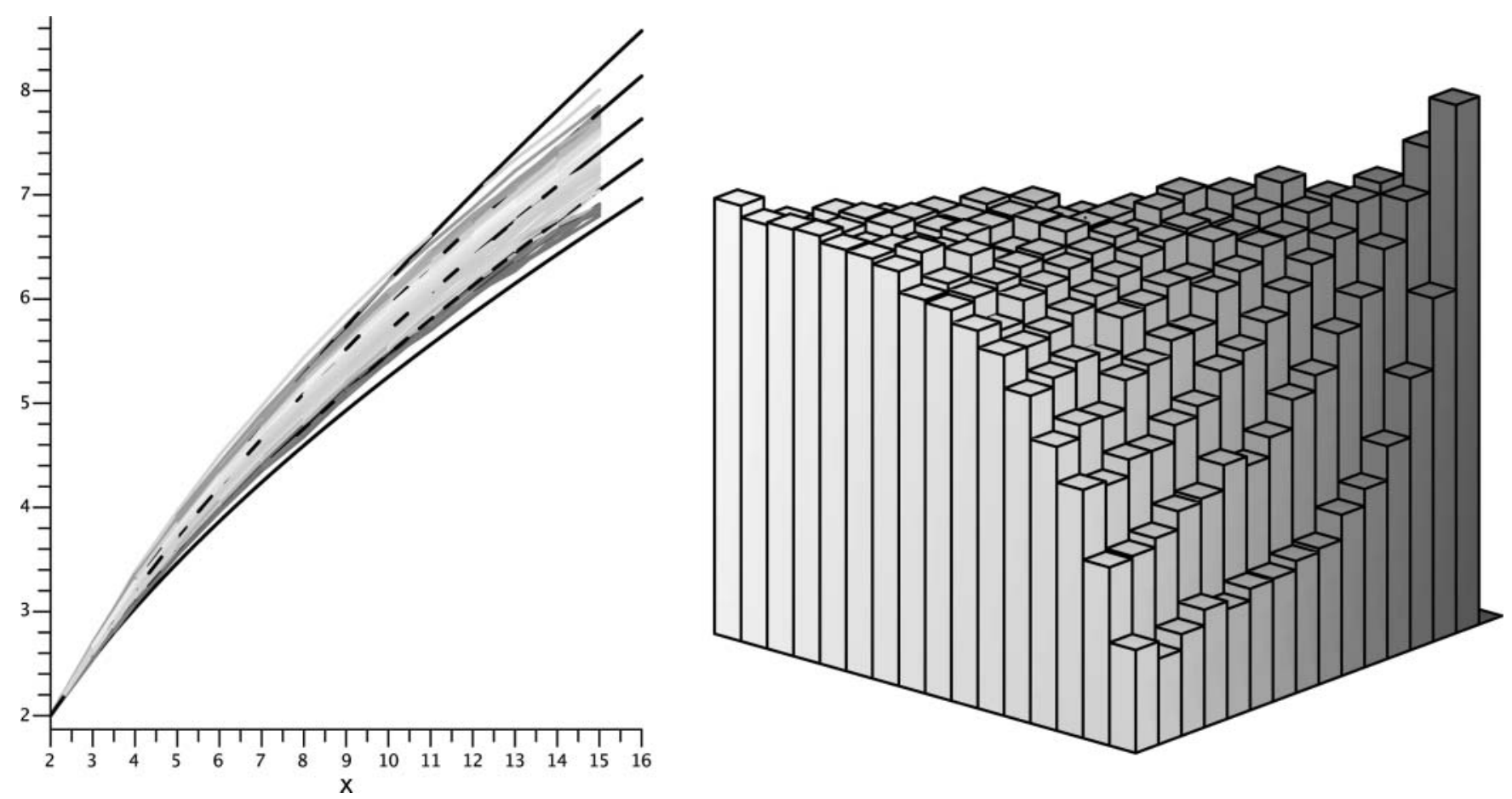

FIGURE 5. Left: Growth of average heights (light gray corresponding to blocks with small "eccentricity" $w / b$, dark gray to large $w / b$ ). The black lines follow growths between $n^{0.6}$ and $n^{0.7}$, equally spaced. Right: Average heights of structures with 13 to 15 blocks by block size (the $b=w=1$ entry has been deleted).

entries will be used to specify what to attach to block 2 . We may use one entry fewer by employing in some systematic way that one position was already used in adding block $i$ for $i>0$.

The procedure is explained in more detail in [Durhuus and Eilers 05] and [Abrahamsen and Eilers 07]. One can prove the following lemma:

Lemma 3.2. If a partition of the $b \times w$ block exists with term and element counts $\mathrm{T}$ and $\mathrm{E}$, then

$$
\# \mathcal{U}_{b \times w}[n]=\left(\begin{array}{c}
2 \boldsymbol{T}+(n-2)(2 \boldsymbol{\top}-1) \\
n-1
\end{array}\right) \mathrm{E}^{n-1}
$$

and

$$
h_{b \times w} \leq \mathrm{E}(2 \mathrm{~T}-2)\left(1-\frac{1}{2 \mathrm{~T}-1}\right)^{-2 \mathrm{~T}+1}
$$

Sketch of proof of Theorem 2.3: There exist partitions with term and element counts as indicated in Figure 6. Applying Lemma 3.2 and the fact that $(1-1 / x)^{-x}$ decreases, one gets the estimates.
Remark 3.3. The values that are especially amenable to Monte Carlo analysis may be characterized as the values for which the estimate above is relatively tight.

\subsection{Monte Carlo Methods}

Since we know the size of $\mathcal{U}_{b \times w}[n]$ from Lemma 3.2, we may use the map $\Psi$ to estimate $T_{b \times w}(n)$, if we take into account the fact that the map is not injective. Hence for each tuple $t$ with $\Psi(t) \in \mathcal{T}_{b \times w}[n]$, we need to determine $\Psi^{-1}(\Psi(t))$, i.e., the number of tuples that are mapped into the same structure as $t$.

Assume that we have randomly created $m$ tuples from $\mathcal{U}_{b \times w}[n]$. Let $t_{1}, \ldots, t_{r}$ be those mapped into $\mathcal{T}_{b \times w}[n]$ by $\Psi$. With

$$
\alpha=\frac{\# \mathcal{U}_{b \times w}[n]}{m} \sum_{k=1}^{r} \frac{1}{\# \Psi^{-1}\left(\Psi\left(t_{k}\right)\right)}
$$

and

$$
\beta=\frac{\left(\# \mathcal{U}_{b \times w}[n]\right)^{2}}{m} \sum_{k=1}^{r} \frac{1}{\left(\# \Psi^{-1}\left(\Psi\left(t_{k}\right)\right)\right)^{2}}-\sqrt{\alpha},
$$

we have the $95 \%$ confidence interval for $T_{b \times w}(n)$,

$$
\left[\alpha-1.96 \sqrt{\frac{\beta}{m}}, \alpha+1.96 \sqrt{\frac{\beta}{m}}\right] .
$$




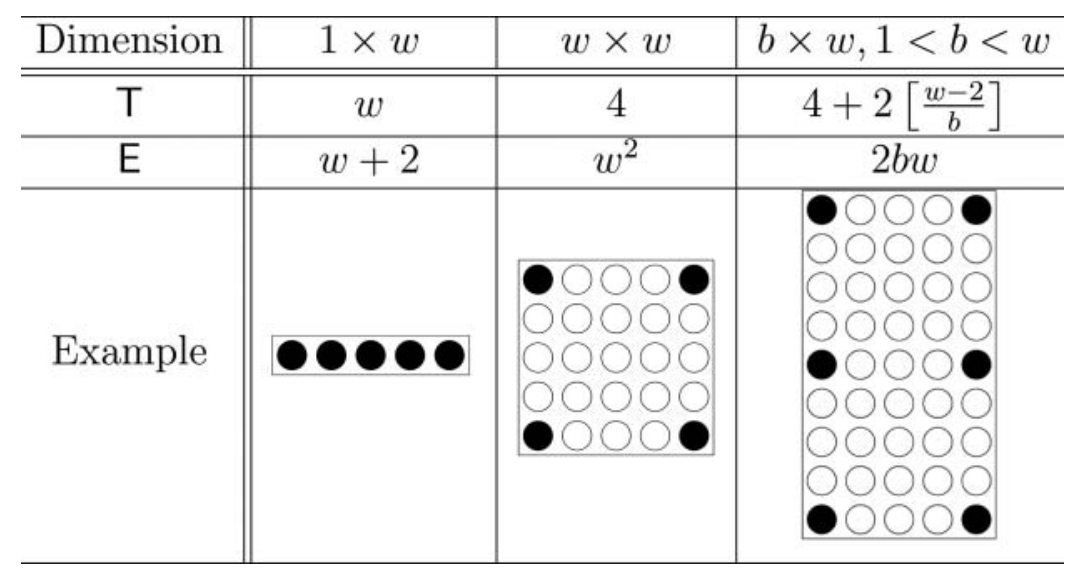

FIGURE 6. Even partitions.

To calculate efficiently the number of tuples that create a given configuration, we define the concept of a characteristic graph. For a given configuration, the characteristic graph is the graph whose vertices are the blocks in the configuration whose vertices are adjacent exactly when the blocks are attached to each other. One easily deduces the following result:

Proposition 3.4. The number of tuples creating $L$ is the number of spanning trees in a characteristic graph $G$ of $L$.

We can use Kirchhoff's matrix tree theorem [Kirchhoff 47], [Matousek 98, p. 323] in calculating the number of spanning trees. Let $G=(V, E)$ be a graph, $V=\left\{v_{1}, \ldots, v_{n}\right\}$. Let $\delta_{i}$ be the degree of $v_{i}$ and let $e_{i j}$ be the number of vertices from $v_{i}$ to $v_{j}$ (since our graph is simple, this number is in $\{0,1\})$. The theorem states that the number of spanning trees is given as $N=\operatorname{det} A[t]$, where $A[t]$ has entries

$$
a_{i j}=\left\{\begin{array}{l}
\delta_{i}, \quad i=j, \\
-e_{i j}, i \neq j,
\end{array}\right.
$$

where $i, j=1, \ldots, n-1$.

Proposition 3.5. When $\Psi(t) \in \mathcal{T}_{b \times w}[n]$, we have

$$
\begin{aligned}
& \# \Psi^{-1}(\Psi(t)) \\
& =\operatorname{det} A[t] \cdot n \cdot 2^{-\delta_{b, w}} \\
& \quad \times \begin{cases}1 / 2 & \text { if } \Psi(t) \text {. symmetric by } 90^{\circ}, \\
1 & \text { if } \Psi(t) \text { symmetric by } 180^{\circ} \\
2 & \text { otherwise. }\end{cases}
\end{aligned}
$$

Example 3.6. Consider the structure shown in Figure 1(c). The characteristic graph is of the form $\boxplus$, which is found by Kirchhoff's theorem to have 192 spanning trees. The structure is symmetric after a rotation by 180 degrees, and therefore the number of tuples corresponding to configurations representing the structure is $192 \cdot 9=1728$.

\section{ACKNOWLEDGMENTS}

We are grateful to colleagues at the Department of Mathematical Sciences at the University of Copenhagen for fruitful discussions, notably Bergfinnur Durhuus, Ernst Hansen, and Niels Richard Hansen, as well as the entire computing department, led by Rasmus Borup Hansen, for their help in keeping our rather demanding processes alive. We also wish to extend our thanks to all the people involved in the LDraw project, from which we have benefited in an obvious way.

\section{REFERENCES}

[Abrahamsen and Eilers 07] M. Abrahamsen and S. Eilers. "Efficient Counting of LEGO Structures." Tech. report, University of Copenhagen, available online (www.math. ku.dk/papers/eclbii.pdf), 2007.

[Durhuus and Eilers 05] B. Durhuus and S. Eilers. "On the Entropy of LEGO." Preprint available from World Wide Web (http://arxiv.org/abs/math/0504039).

[Flajolet and Sedgewick 08] P. Flajolet and R. Sedgewick. Analytic Combinatorics. Cambridge, UK: Cambridge University Press, 2008. 
[Kirchhoff 47] H. Kirchhoff. "Über die Auflösung der Gleichungen, auf welche man bei der Untersuchung der linearen Verteilung galvanischer Ströme geführt wird." Ann. Phys. Chem. 72 (1847), 497-508.

[Madras 95] N. Madras, "A Rigorous Bound on the Critical Exponent for the Number of Lattice Trees, An- imals and Polygons." J. Stat. Phys. 78 (1995), 681699 .

[Matousek 98] J. Matousek. Invitation to Discrete Mathematics. Oxford: Oxford University Press, 1998.

Mikkel Abrahamsen, Department for Mathematical Sciences, University of Copenhagen, Universitetsparken 5, DK2100 Copenhagen $\varnothing$, Denmark (Mikkel.A@math.ku.dk)

Søren Eilers, Department for Mathematical Sciences, University of Copenhagen, Universitetsparken 5, DK-2100 Copenhagen Ø, Denmark (eilers@math.ku.dk)

Received May 25, 2008; accepted August 8, 2010. 